\title{
Validade e confiabilidade da versão brasileira da Center for Epidemiological Scale - Depression (CES-D) em idosos brasileiros
}

\author{
Samila Sathler Tavares Batistoni ${ }^{1}$ - Universidade de São Paulo, São Paulo, Brasil \\ Anita Liberalesso Néri - Universidade Estadual de Campinas, Campinas, Brasil \\ Ana Paula Cupertino - University of Kansas Medical Center, Kansas City, Estados Unidos
}

\begin{abstract}
Resumo
Foram identificados indicadores de validade de construto e de confiabilidade interna para a Center for Epidemiological ScaleDepression (CES-D) aplicada a idosos recrutados na comunidade, em um estudo que envolveu duas medidas sucessivas. A amostra foi composta de 347 idosos ( $73 \%$ mulheres) com idade média de 71,96 anos $(D P=8,45)$, os quais foram submetidos à versão brasileira da CES-D em duas ocasiões, com um tempo médio de 15,7 meses $(D P=4,57)$ entre as medidas. Os dados da segunda medida da CES-D foram submetidos à análise fatorial confirmatória, que revelou adequação do ajuste do modelo anterior com três fatores ("afetos negativos", "dificuldades de iniciar comportamentos", "afetos positivos"). O índice de consistência interna foi 0,82 para a escala, 0,77 para o fator $1,0,59$ para o fator 2 e 0,47 para o fator 3 . A prevalência de depressão foi a mesma nas duas medidas (34\%).

Palavras-chave: Depressão, Idosos, Psicometria.
\end{abstract}

\section{Validity and reliability of the Brazilian version of the Center for Epidemiological Scale - Depression (CES-D) in Brazilian elderly}

\begin{abstract}
Construct validity and internal reliability concerning the Center for Epidemiological Study-Depression (CES-D) were described in a prospective study with elderly people living in the community. The sample consisted of 347 elderly adults $(73 \%$ women), 71.96 years old in average $(\mathrm{SD}=8.45)$, which were submitted to two measures of depressive symptoms through the CES-D. The mean time between measures was 15.7 months $(\mathrm{SD}=4.57)$. Confirmatory factorial analysis performed on the second measure revealed the same factors identified in the first measure ("negative affection", "difficulty in initiating behaviors" and "positive affection"). Cronbach alpha was 0.82 for the scale, and $0.77,0.59$ and 0.47 , respectively for factors 1,2 , and 3 . Prevalence of depressive symptoms was consistent in both measures $(34 \%)$.

Keywords: Depression, Elderly, Psychometrics.
\end{abstract}

A depressão é um construto psicopatológico não avaliado diretamente por medidas objetivas. Sua identificação depende de relatos de comportamentos e sintomas que teoricamente são considerados como integrantes de uma síndrome. Inventários e escalas que permitem registrar a presença e a frequência de sintomas depressivos foram construídos com o objetivo de rastrear, identificar e avaliar a intensidade e a forma de apresentação desses sintomas. A maioria foi construída e conta com indicadores de confiabilidade e validade em amostras clínicas, ou seja, para indivíduos que buscam serviços de saúde com uma queixa psicopatológica identificada. São menos numerosos os instrumentos especificamente construídos para rastrear e medir sintomas

\footnotetext{
1 Agradecimentos às fontes de auxílio à pesquisa: CNPq - Profix (processo $\mathrm{n}^{\circ}$ 540956-01/5NV); Capes - Coordenação de Aperfeiçoamento de Pessoal de Nível Superior - Demanda Social; Endereço para correspondência:

Curso de Graduação em Gerontologia/Escola de Artes, Ciências e Humanidades - USP - Av. Arlindo Bettio, 1000 - Ermelino Matarazzo - 03828-000 - São Paulo-SP

E-mail: samilabatistoni@usp.br
}

depressivos em populações recrutadas ou provenientes da comunidade.

Entre os instrumentos planejados para a população não-clínica figura a Center for Epidemiological Studies-Depression (CES-D). Elaborada pelo National Institute of Mental Health (EUA), esta escala de autorrelato visa identificar humor depressivo em estudos populacionais (Radlof, 1977). Surgiu da reunião dos inventários de depressão de Zung (Zung's depression scale Zung, 1965), do Inventário de depressão de Beck (Beck's Depression Inventory - Beck, Ward, Mendelson, Mock \& Erbaugh, 1961), do Inventário de Raskin (Raskin, Schulterbrandt \& Reating, 1967) e do Inventário de Personalidade MMPI (Minnesota Multiphasic Personality Inventory Dahlstron \& Welsh, 1960). Em estudos populacionais, a CES-D e o Inventário de Depressão de Beck são os instrumentos mais utilizados (Maj \& Sartorius, 2005).

A CES-D inclui os principais componentes dos transtornos depressivos conforme os critérios diagnósticos do DSM-IV. Trata-se de uma escala de 20 itens de 0 a 3 pontos cada um, que comportam avaliação da frequência de sintomas 
depressivos vividos na semana anterior à entrevista (nunca, poucas vezes, maioria das vezes, sempre). Incluem questões sobre o humor, sintomas somáticos, interações com os outros e funcionamento motor. No estudo original da estrutura fatorial da CES-D de Radlof (1977), quatro fatores foram identificados: (a) humor depressivo (incluindo itens como tristeza, medo e sentimentos de vazio); (b) afetos positivos (como felicidade, sentir-se bem, aproveitar a vida e esperança - itens que têm sua pontuação invertida na contagem do escore total); (c) sintomas somáticos (como cansaço, esforço e dificuldade de concentração); (d) problemas interpessoais (não sentir-se amado, sentir que as pessoas não têm se mostrado amistosas).

Desenvolvida no contexto norteamericano, a CES-D já foi traduzida e validada para uso em diferentes culturas. De maneira geral, os estudos de validação em diferentes nações, grupos étnicos e grupos etários mostram que a escala se correlaciona significativamente com indicadores clínicos de depressão e que tem bons índices de confiabilidade interna (entre 0,8 e 0,9 ), de estabilidade ou reprodutibilidade, de validade de construto, que sugerem ser útil como instrumento de rastreio de sintomas depressivos.

Além de servir para identificar as características do instrumento em si, o exame das propriedades psicométricas da CES-D é útil à compreensão do próprio construto de depressão em diferentes populações. Estratégias de análise fatorial têm possibilitado identificar equivalência ou diferença entre as variáveis latentes que compõem o construto depressão e compreender quais fatores respondem pela presença dessa síndrome nos grupos pesquisados em diferentes culturas.

Silveira e Jorge (2000) utilizaram a CES-D traduzida e adaptada semanticamente para o Brasil e descreveram sua confiabilidade e sua validade de construto por meio de análise fatorial, em amostra de adolescentes e adultos jovens. A consistência interna da versão brasileira da CES-D entre populações jovens (clínica e não-clínica) medida pelo alfa de Cronbach, foi $>0,84$. Essa análise também revelou que duas questões poderiam ser excluídas da escala por apresentarem baixa correlação com todas as demais ("tive pouco apetite"; "senti-me tendo tanto valor quanto outras pessoas"). A análise fatorial resultou em uma solução de quatro fatores explicativos de $53,8 \%$ da variância, resultado similar aos encontrados em estudos internacionais envolvendo a população geral. Os fatores encontrados por Silveira e Jorge (2000) foram semelhantes às subescalas propostas por Radlof (1977) e assim não foram reinterpretadas.

A CES-D superestima a depressão em idosos pelo fato de incluir sintomas somáticos, naturalmente mais presentes nessa população. Por exemplo, Williamson e Schulz (1992) encontraram associação entre piores condições de saúde física objetiva e subjetiva e altos escores na CES-D em idosos da comunidade. Outros autores acreditam que, controladas as condições de fragilidade, os idosos não são mais vulneráveis à depressão do que outros grupos de idade (Blazer, 2003). Reynolds (1994) aponta que depressão entre idosos se apresenta com notável heterogeneidade em razão do fator etário. Assim, defende ser melhor que o diagnóstico clínico tenha um enfoque superinclusivo, levando em conta e analisando todos os sintomas existentes, antes de descartá-los ou de atribuí-los a outras causas. Estudos sobre a apresentação clínica da depressão entre idosos revelam que entre esses seriam mais comuns os sintomas somáticos ou hipocondríacos, a baixa frequência de antecedentes familiares de depressão e a pior resposta ao tratamento (Gatz, Kasl-Godley \& Karel, 1996). Segundo Reifler (1994), de modo geral os idosos queixam-se mais de perda de interesse pelos acontecimentos que os cercam, de fadiga, de despertar muito cedo pela manhã e não voltar a dormir, de falta de memória, de pensamentos sobre morte e de desesperança geral. Idosos queixam-se menos de humor deprimido e de outros sintomas disfóricos essenciais ao diagnóstico de depressão maior do que as populações jovens.

Descrever o desempenho de idosos em escalas de rastreio de depressão que incluem sintomas somáticos (como a CES-D) pode auxiliar na compreensão das diferenças etárias no autorrelato de sintomas trazendo implicações clínicas importantes. Em estudo com 903 idosos brasileiros residentes na comunidade, Batistoni, Neri e Cupertino (2007) verificaram que a estrutura do construto depressão gerada por análise fatorial exploratória dos itens diferiu da estrutura identificada no estudo de Silveira e Jorge (2000) com amostra de jovens brasileiros e também diferiu da estrutura original de Radlof (1977). No estudo brasileiro, os itens ligados a conteúdos volitivos e somáticos corresponderam ao segundo fator de explicação da variância das respostas à CES-D, enquanto na versão original eles aparecem no fator três. 
Análises fatoriais confirmatórias têm mostrado boa reprodutibilidade das medidas da escala, porém confirmando diferenças na estrutura e na hierarquia dos quatro fatores da CES-D em diferentes grupos etários, culturais, de renda e escolaridade, assim como em grupos afetados por eventos estressantes e por suporte social insatisfatório (Chiriboga, Jang, Banks \& Kim, 2007). Por exemplo, os itens que compõem o fator "afetos negativos" parecem ser mais reativos a atributos internos, tais como personalidade e condições de saúde, enquanto os itens relativos a "afetos positivos" parecem ser mais responsivos a eventos de vida externos, tais como os ligados ao envolvimento social (Clark \& Watson, 1991). Zich, Atkinson e Greenfield (1990) relataram que a baixa pontuação de idosos nos itens do fator "afetos positivos" associou-se com redes do suporte social deficitárias e com falta de experiências positivas.

Nesse sentido, o presente artigo buscou dar continuidade ao estudo psicométrico da CES$\mathrm{D}$ em idosos, trabalho já iniciado com uma publicação em periódico de 2007 (Batistoni, Neri \& Cupertino, 2007), o qual levantou indicadores de validade de construto e de critério tomando como padrão de comparação a versão brasileira da Geriatric Depression Scale - GDS (Almeida \& Almeida, 1999; Yesavage \& cols., 1983). No presente trabalho, foram investigadas evidências de reprodutibilidade da estrutura fatorial da CESD obtida por Batistoni, Neri e Cupertino (2007) por meio de análise fatorial confirmatória aplicada a uma medida sequencial de sintomas depressivos em idosos residentes na comunidade.

\section{Método}

\section{Participantes}

Os idosos integram o banco de dados da primeira e segunda medida do Projeto Estudo do Envelhecimento Saudável (PENSA), para o qual foram selecionados pelo método de amostragem sistemática. O estudo PENSA foi coordenado pela profa. dra. Ana Paula Fabrino Bretas Cupertino e desenvolvido na Universidade Federal de Juiz de Fora - MG, com auxílio financeiro do Programa de Fixação de Doutores do CNPq - PROFIX (processo no 540956/01-5). O projeto de pesquisa teve aprovação do Comitê de Ética em Pesquisas com Seres Humanos do Hospital Universitário da Universidade Federal de Juiz de Fora, sob processo $n^{\circ} 170-009 / 2002$. Recrutadores treinados visitaram todos os domicílios dos bairros da cidade de Juiz de Fora, MG, cujo percentual de idosos (60 anos e mais) residentes era superior a 15\% (14 bairros), segundo dados do Censo 2000 do Instituto Brasileiro de Geografia e Estatística (IBGE, 2000). Foram visitados 7.089 domicílios, dentre os quais foram identificados $1.686 \mathrm{com}$ pelo menos um idoso. Novecentos e cinquenta e seis idosos aceitaram participar do estudo (56\%) e assinaram um Termo de Consentimento Livre e Esclarecido, 614 recusaram (36\%) e 116 (8\%) pareceram física ou cognitivamente incapacitados. Cada entrevista foi realizada numa única sessão, de aproximadamente duas horas, envolvendo itens de autorrelato, de natureza sociodemográfica, física, psicológica e sociológica. Os dados da primeira onda de medidas (Tempo 1 ou T1) foram coletados entre 2002 e 2003. Entre 2003 e 2004, após um período médio de 15,7 meses $(\mathrm{DP}=4,57)$ foi realizada uma medida de autorrelato de saúde, sintomas depressivos e cognição com um terço da amostra inicial de idosos $(n=347)$, selecionados por sorteio simples do banco de dados do PENSA (Tempo 2 ou T2). Os dados desta segunda medida foram utilizados no presente estudo e envolveram a presença de $73,5 \%$ de idosos do sexo feminino e a idade média foi de 71,9 anos $(\mathrm{DP}=8,45)$, sendo $45,8 \%$ de 60 a 69 anos, $34,8 \%$ de 70 a 79 anos e $19,3 \%$ mais de 80 anos. No que se referiu à escolaridade, cerca de 3\% eram analfabetos, $45 \%$ eram alfabetizados ou possuíam apenas o primário completo, $17,5 \%$ possuíam o ginasial, $24 \%$ alcançaram o ginasial e $11 \%$ tinham curso superior completo ou incompleto.

\section{Instrumento}

Escala de rastreio de sintomas depressivos CES-D - Center for Epidemiological Studies Depression (Radlof, 1977), composta por 20 itens, que comportam avaliação da frequência de sintomas depressivos vividos na semana anterior à entrevista $(0=$ nunca ou raramente, $2=$ às vezes, $3=$ maioria das vezes ou sempre). O escore final varia de 0 a 60 pontos; os itens $4,8,12$ e 16 são pontuados em ordem decrescente. Originalmente, o ponto de corte da escala CES-D para identificar presença de sintomas depressivos é de $\geq 16$ pontos. Em amostras norte-americanas não-idosas a consistência interna alcançou de 0,8 a 0,9. Em estudo de validação com idosos brasileiros, Batistoni, Neri e Cupertino (2007) identificaram o ponto de corte $\geq 12$ pontos para identificar possíveis casos de depressão. Nesse mesmo estudo foi encontrado alto índice de consistência interna $(\alpha=0,86)$ e uma solução fatorial de 3 fatores que 
explicaram $47,5 \%$ da variabilidade total dos dados (1- afetos negativos; 2- dificuldade de iniciar comportamentos; 3 - afetos positivos).

\section{Análise de dados}

Os dados relativos às respostas dos 347 idosos à CES-D na medida de seguimento (Tempo 2) foram submetidos à análise fatorial confirmatória com o intuito de verificar a reprodutibilidade da estrutura fatorial obtida na medida de linha de base (Tempo 1). Para testar a hipótese da composição fatorial da CES-D utilizou-se a análise fatorial confirmatória, modelo de equações estruturais para variáveis latentes (structural equation modeling for latent variables). Este modelo é composto de parâmetros fixos (cargas fatoriais iguais a zero) e parâmetros livres a serem estimados (cargas fatoriais diferentes de zero). São calculadas estatísticas para testar a adequação do ajuste do modelo (goodness of fit) e verificar se os fatores explicam as correlações observadas entre as variáveis, conforme o modelo teórico proposto. A estrutura fatorial da CES-D na medida de linha de base, que foi submetida a teste, aparece na Tabela 1.

Tabela 1 - Estrutura fatorial da CES-D resultante da análise de componentes principais da matriz reduzida para a amostra total do PENSA na primeira medida do estudo

\begin{tabular}{|c|c|}
\hline Fator $1-\mathrm{EV}=5,742-\mathrm{VE}=28,7 \%$ & Cargas \\
\hline 6 - senti-me deprimido & 0,726 \\
\hline 18 - senti-me triste & 0,708 \\
\hline 19 - senti que as pessoas não gostavam de mim & 0,597 \\
\hline 17 - tive crises de choro & 0,591 \\
\hline 09 - considerei que a vida tinha sido um fracasso & 0,591 \\
\hline 14 - senti-me sozinho & 0,552 \\
\hline 20 - não consegui levar adiante minhas coisas & 0,458 \\
\hline 10 - senti-me amedrontado & 0,438 \\
\hline 13 - falei menos do que o habitual & 0,392 \\
\hline Dificuldades de iniciar comportamentos & \\
\hline 7 - senti que tive que fazer esforço para fazer tarefas habituais & 0,613 \\
\hline 2 - não tive vontade de comer, pouco apetite & 0,612 \\
\hline 1 - senti-me incomodado com coisas que habitualmente não me incomodam & 0,604 \\
\hline $\begin{array}{l}3 \text { - senti não conseguir melhorar meu estado de ânimo, mesmo com ajuda de familiares e } \\
\text { amigos }\end{array}$ & 0,559 \\
\hline 5 - senti dificuldades em me concentrar no que fazia & 0,557 \\
\hline Fator $3-\mathrm{EV}=1,183-\mathrm{VE}=5,9 \%$ & \\
\hline 8 - senti-me otimista sobre o futuro & 0,719 \\
\hline 16 - aproveitei minha vida & 0,645 \\
\hline 4 - senti-me, comparando-me às outras pessoas, tendo tanto valor quanto a maioria delas & 0,616 \\
\hline 12 - estive feliz & 0,600 \\
\hline Fator $4-\mathrm{EV}=1,08-\mathrm{VE}=5,4 \%$ & \\
\hline 15 - as pessoas não foram amistosas comigo & 0,761 \\
\hline 11 - meu sono não foi repousante & 0,507 \\
\hline
\end{tabular}

As principais estatísticas e os parâmetros utilizados na análise fatorial confirmatória foram: Teste Qui-Quadrado para Adequação de Ajuste: (verifica se a matriz de covariâncias estimada é igual à matriz de covariâncias da amostra - nível de significância maior que 0,05); Razão QuiQuadrado $\left(\chi^{2} / \mathrm{gl}\right)$ : valor menor que 2.0 para indicar bom ajuste; GFI (Goodness of Fit Index): valor de aceitação maior ou igual a 0,85 ; AGFI (GFI Adjusted for Degrees of Freedom): valor de aceitação maior ou igual a 0,80; RMR (Root Mean
Square Residual): valor de aceitação menor ou igual a 0,10; CFI (Bentler's Comparative Fit Index): valor de aceitação maior ou igual a 0,90; NNFI (Bentler \& Bonett's Non-normed Index): valor de aceitação maior ou igual a 0,90 .

Para analisar a qualidade do ajuste dos dados aos fatores propostos foram feitos testes de significância para as cargas fatoriais (valores de t>1.96 indicam que $\mathrm{o}$ item tem uma carga significativa dentro do fator). Para propor modificações nos fatores, mediante a exclusão de 
alguns itens de determinados fatores, foi utilizado o teste de Wald. Também foi feito o teste do Multiplicador de Lagrange, que define a necessidade de realocação de um item para outro fator, a fim de melhorar a correlação entre os itens dentro do mesmo fator. Semelhantemente ao teste de Wald, ele mostra o quanto haverá de modificação na estatística qui-quadrado ao se realocar um dado item num novo fator. Os dados categóricos sobre sintomatologia depressiva foram tratados com os testes de Mc Nemar e os escalares com o teste de Wilcoxon, ambos para comparar as médias de pontuação dos idosos na CES-D nos tempos (T1 e T2) e para comparar as diferenças na prevalência de sintomas depressivos rastreados pela CES-D.

\section{Resultados}

Não houve diferença significativa quanto à pontuação média na CES-D nas duas medidas (T1: média $=11 ; D P=10,18 ;$ T2: média $=10,2 ; D P=8,61$; $p=0,061)$. Também não houve diferença estatisticamente significante entre a prevalência de depressão nas duas medidas do estudo (em T1: 33,8\%; em T2: 34,8\%; $p=0,753)$. As Tabelas 2, 3 e 4 apresentam os resultados da análise fatorial confirmatória para os 18 itens da escala CES-D (foram desconsiderados os itens 11 e 15 do $4^{\circ}$ fator, não foi interpretado na primeira análise fatorial).

Tabela 2 - Índices de adequação do modelo inicial testado na análise fatorial confirmatória da CES-D*

\begin{tabular}{lc}
\hline Índice & Modelo inicial \\
\hline$\chi^{2}$ (qui-quadrado) & 214,7865 (gl=132); p-valor=0,0001 \\
$\chi^{2} /$ gl (razão qui-quadrado) & 1,6272 \\
GFI (goodness of fit index) & 0,9388 \\
AGFI (gfi adjusted for degrees of freedom) & 0,9208 \\
RMR (root mean square residual) & 0,0418 \\
CFI (bentler's comparative fit index) & 0,9336 \\
NNFI (bentler \& bonett's (1980) non-normed index) & 0,9230 \\
\hline
\end{tabular}

* número de itens da CES-D: 18 itens; $\mathrm{n}=347$ sujeitos.

Tabela 3 - Resultado da análise fatorial confirmatória para o modelo inicial testado

\begin{tabular}{lcc}
\hline Itens da CES-D & Cargas & $\mathrm{t}$ \\
\hline Fator 1 & 0,763 & $15,29 *$ \\
6- senti-me deprimido & 0,781 & $20,73^{*}$ \\
18- senti-me triste & 0,207 & $5,72^{*}$ \\
19- senti que as pessoas não gostavam de mim & 0,038 & $10,89^{*}$ \\
17- tive crises de choro & 0,345 & $9,30^{*}$ \\
9- considerei que minha vida tinha sido um fracasso & 0,459 & $9,61^{*}$ \\
14- senti-me sozinho/a & 0,376 & $9,11^{*}$ \\
20- não consegui levar adiante minhas coisas & 0,391 & $8,68^{*}$ \\
10- senti-me amedrontado & 0,439 & $9,27^{*}$ \\
13- falei menos do que o habitual & \\
Fator 2 & 0,571 & $9,86^{*}$ \\
7- senti que tive que fazer esforço para dar conta das minhas tarefas habituais & 0,270 & $5,94^{*}$ \\
2- não tive vontade de comer, ou seja, tive pouco apetite & 0,392 & $6,29 *$ \\
1- senti-me incomodado com coisas que normalmente não me incomodam & 0,568 & $10,0^{*}$ \\
3- senti não conseguir melhorar meu estado de ânimo mesmo com a ajuda de amigos e e & \\
familiares & 0,414 & $7,55^{*}$ \\
5- senti dificuldade em me concentrar no que estava fazendo & \\
Fator 3 & 0,400 & $5,09^{*}$ \\
8- senti-me otimista sobre o futuro & 0,957 & $13,26^{*}$ \\
16- aproveitei minha vida & 0,007 & 0,09 \\
4- senti-me, comparando-me às outras pessoas, tendo tanto valor quanto à maioria delas & 0,649 & $11,52^{*}$ \\
12- estive feliz &
\end{tabular}

* valores significativos das cargas dos itens $(\mathrm{p}<0,05)$ para t-value $>1,96$. 
A maioria dos critérios de adequação de ajuste obteve valor ideal para aceitação do modelo teórico proposto. Pela estimativa das cargas dos fatores (Tabela 5) verifica-se que todos os itens foram significativos, exceto o item 4 do fator 3 . Foi feito o Teste de Wald para identificar qual item da CES-D poderia ser excluído sem alterar significativamente a estrutura fatorial. Nesse teste o valor de $\mathrm{p}$ deve se aproximar de 1 . O teste identificou que a retirada do item $4\left(\chi^{2}=0,008\right.$ $\mathrm{gl}=1 ; \quad p=0,927)$ não afeta significativamente a estatística qui-quadrado. Além de indicar a retirada do item 4 do fator 3, a Análise dos Multiplicadores de Lagrange (Tabela 4) indica a realocação do item 1 no fator 1 ; dos itens 6,8 e 16 no fator 2 ; e dos itens 17 e 19 no fator 3. O item 17 também poderia ser realocado para outro fator, mas, geralmente, opta-se pelo maior multiplicador de Lagrange.

Tabela 4 - Realocação de itens nos fatores segundo os resultados da análise fatorial confirmatória da CES$\mathrm{D}$

\begin{tabular}{cccc}
\hline Item & Fator & $\chi^{2}$ & p-valor \\
\hline 17 & 3 & 10,04 & 0,0015 \\
6 & 2 & 9,76 & 0,0018 \\
17 & 2 & 9,24 & 0,0024 \\
1 & 1 & 6,99 & 0,0082 \\
8 & 2 & 6,23 & 0,0125 \\
16 & 2 & 5,25 & 0,0219 \\
19 & 3 & 4,23 & 0,0396 \\
\hline
\end{tabular}

Após a realocação dos itens nos fatores sugerida pelos multiplicadores de Lagrange e depois da exclusão do item 4 sugerida pelo teste de Wald, a análise fatorial confirmatória da CES-D foi refeita (Tabelas 5, 6 e 7). A maioria dos critérios de adequação de ajuste obteve valor ideal para aceitação do modelo teórico proposto, embora com uma pequena queda nos valores dos índices de ajuste do modelo, o que privilegiaria o $1^{\circ}$ modelo. A Tabela 6 mostra que todos os itens foram significativos na estimação das cargas dos fatores do modelo reespecificado.

Tabela 5 - Índices de adequação do ajuste do modelo reespecificado após primeira realocação dos itens *

\begin{tabular}{lc}
\hline Índice & Modelo reespecificado \\
\hline$\chi^{2}$ (qui-quadrado) & $228,6166(\mathrm{gl}=116) ; \mathrm{p}$-valor=0,0001 \\
$\chi^{2} / \mathrm{gl}$ (razão qui-quadrado) & 1,9708 \\
GFI (goodness of fit index) & 0,9295 \\
AGFI (gfi adjusted for degrees of freedom) & 0,9071 \\
RMR (root mean square residual) & 0,0461 \\
CFI (bentler's comparative fit index) & 0,9099 \\
NNFI (bentler \&o bonett's (1980) non-normed index) & 0,8943 \\
\hline
\end{tabular}

* Número de itens da CES-D: 17 itens; $\mathrm{n}=347$ sujeitos.

Tabela 6 - Resultado da análise fatorial confirmatória para o modelo reespecificado, após primeira realocação dos itens (continua)

\begin{tabular}{lcc}
\hline Itens da CES-D & Cargas & $\mathrm{t}$ \\
\hline Fator 1 & & \\
18- senti-me triste & 0,786 & $15,97^{*}$ \\
9- considerei que minha vida tinha sido um fracasso & 0,037 & $9,55^{*}$ \\
14- senti-me sozinho/a & 0,448 & $9,35^{*}$ \\
20- não consegui levar adiante minhas coisas & 0,373 & $9,02^{*}$ \\
10- senti-me amedrontado & 0,390 & $8,67^{*}$ \\
13- falei menos do que o habitual & 0,442 & $9,37^{*}$ \\
1- senti-me incomodado com coisas que normalmente não me incomodam & 0,386 & $6,55^{*}$
\end{tabular}


Tabela 6 - Resultado da análise fatorial confirmatória para o modelo reespecificado, após primeira realocação dos itens (conclusão)

Itens da CES-D

Fator 2

7- senti que tive que fazer esforço para dar conta das minhas tarefas habituais

2- não tive vontade de comer, ou seja, tive pouco apetite

$0,5389,77 *$

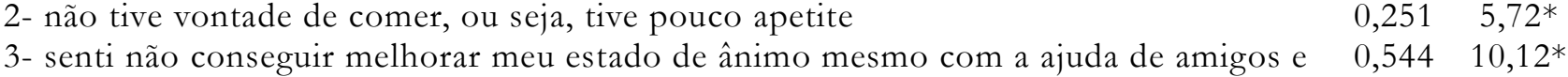

familiares

5- senti dificuldade em me concentrar no que estava fazendo

6- senti-me deprimido

8- senti-me otimista sobre o futuro

16- aproveitei minha vida

$0,391 \quad 7,42 *$

$0,777 \quad 15,35^{*}$

$0,2753,65^{*}$

Fator 3

$0,771 \quad 11,55^{*}$

19- senti que as pessoas não gostavam de mim

$0,174 \quad 5,16^{*}$

17 - tive crises de choro

$0,317 \quad 7,21 *$

12- estive feliz

$0,4337,18^{*}$

* Valores significativos das cargas dos itens $(\mathrm{p}<0,05)$ para $\mathrm{t}-\mathrm{value}>1,96$.

Para esse modelo, o Teste de Wald não indicou da retirada de nenhum dos itens. Já a Análise dos Multiplicadores de Lagrange (Tabela 6) sugeriu a realocação dos itens 5, 6 e 17 ao fator
1; do item 19 ao fator 2; e dos itens 1 e 3 ao fator 3. No entanto, como os valores dos índices ficaram piores que os do ajuste anterior, decidiu-se não utilizar a $2^{\mathrm{a}}$ realocação proposta.

Tabela 7 - Segunda realocação de itens nos fatores conforme os resultados da análise fatorial confirmatória da CES-D

\begin{tabular}{cccc}
\hline Item & Fator & $\chi^{2}$ & p-valor \\
\hline 6 & 1 & 13,43 & 0,0002 \\
5 & 1 & 12,68 & 0,0004 \\
17 & 1 & 7,22 & 0,0072 \\
19 & 2 & 5,24 & 0,0220 \\
1 & 3 & 4,07 & 0,0434 \\
3 & 3 & 3,91 & 0,0480 \\
\hline
\end{tabular}

A seguir, são apresentados os coeficientes de consistência interna ( $\alpha$ de Cronbach) da CES-D na $2^{a}$ avaliação. Índices $\alpha \geq 0,8$ indicam alta consistência interna dos itens e índices $\alpha \geq 0,6$ indicam moderada consistência.

Tabela 8 - Análise da consistência interna da CES-D (N=347)

\begin{tabular}{|c|c|c|c|c|c|}
\hline $\begin{array}{c}\text { Escala Total e } \\
\text { fatores da } \\
\text { CES-D }\end{array}$ & $\begin{array}{l}\text { No de } \\
\text { itens }\end{array}$ & Coeficientes* & $\begin{array}{l}\text { Itens com menor } \\
\text { consistência }\end{array}$ & $\begin{array}{c}\text { Correlação com o } \\
\text { total }\end{array}$ & $\begin{array}{c}\text { Coeficiente* } \\
\text { (após } \\
\text { retirada } \\
\text { dos itens) }\end{array}$ \\
\hline Escore total & 20 & 0,823 & item 4 & $-0,007$ & 0,834 \\
\hline Fator 1 & 9 & 0,771 & - & - & - \\
\hline Fator 2 & 5 & 0,594 & - & - & - \\
\hline Fator 3 & 4 & 0,470 & item 4 & 0,022 & 0,614 \\
\hline
\end{tabular}

* Coeficiente alfa de Cronbach para escala CES-D.

A Tabela 8 permite avaliar que a escala como um todo $\mathrm{e}$ o fator 1 alcançaram alta consistência interna. Os itens dos fatores 2 e 3 não alcançaram altas correlações. Ambos são relativamente pouco explicativos da variância dos dados, conforme já mostrado anteriormente na Tabela 1. 


\section{Discussão}

Os procedimentos de análise fatorial confirmatória demonstraram a reprodutibilidade da estrutura fatorial gerada anteriormente por meio de análise fatorial exploratória. $\mathrm{Na}$ estrutura de três fatores que se confirmou, o primeiro fator ("afetos negativos") manteve-se explicando a maior parte da variabilidade dos dados. Esse fator reúne os itens mais representativos dos sintomas essenciais dos transtornos depressivos (humor deprimido e perda de prazer em atividades) e distingue-se do fator composto por itens de caráter volitivo e somático (denominado de "dificuldades de iniciar comportamentos"), ou de itens que refletem bem-estar, satisfação ou "afetos positivos", estes opostos aos itens do primeiro fator.

A estrutura fatorial original da CES-D, gerada em sua validação para populações adultas jovens (Radlof, 1977), contém quatro fatores: afetos depressivos, afetos positivos, queixas somáticas e sintomas interpessoais. Entretanto, apesar de comporem o construto depressão, essa estrutura fatorial não se repete em diferentes grupos culturais, étnicos e etários (Chapleski, Lamphere, Kaczynski, Lichtenberg \& Dwyer, 1997). Os sintomas volitivos e somáticos comportam-se de forma diferente entre idosos, apresentando-se sozinhos, como um segundo fator na explicação da variabilidade, ou misturando-se a itens de afetos depressivos. O presente estudo confirma esse dado, visto que esse conjunto de sintomas foi o segundo fator explicativo da variância dos dados. No estudo de Silveira e Jorge (2000) com jovens e adolescentes brasileiros, a maioria dos itens correspondentes aos aspectos volitivos e somáticos agruparam-se no terceiro fator, diferentemente do que ocorreu com os idosos do presente trabalho. Esse dado é sugestivo de que os sintomas volitivos e somáticos são mais explicativos da depressão entre idosos do que entre jovens.

Os sintomas interpessoais (quarto fator da estrutura original de Radlof, 1977) não se mantêm como um fator específico entre idosos, ou pela falta de variabilidade das respostas, ou por estarem associados a itens indicativos de afetos depressivos (Davey, Halverson Jr., Zonderman \& Costa Jr., 2004). Krause, Liang e Yatomi (1989) também testaram a estrutura de Radlof e encontraram uma estrutura de três fatores entre idosos, sendo que os sintomas interpessoais apresentaram médias e variâncias mais baixas. $\mathrm{Na}$ análise fatorial exploratória realizada para gerar o modelo fatorial testado nesse estudo, um sintoma interpessoal bem característico (item 15) foi mantido no quarto fator, que não foi interpretado em razão de seu baixo poder explicativo. Por outro lado, outro sintoma interpessoal característico (item 19) apareceu no fator 1 (afetos negativos, dimensão equivalente ao denominado "afetos depressivos" nos estudos internacionais), tendência essa ressaltada por Davey e cols. (2004). No estudo de Silveira e Jorge (2000), os itens interpessoais aparecem no segundo fator, o que sugere serem mais relevantes à explicação da depressão entre jovens e adolescentes do que entre idosos. Há diferenças adicionais entre os grupos de jovens e idosos. No presente trabalho, o fator "afetos positivos" manteve-se como terceiro fator explicativo da variância. No estudo de Silveira e Jorge (2000) com jovens brasileiros, a dimensão "bem-estar", equivalente a "afetos positivos", foi o quarto fator explicativo da variância.

Outro indicador de confiabilidade da CES$D$ entre idosos foi dado pelo índice de consistência interna da escala na segunda medida realizada (T2). A psicometria preconiza que, via de regra, instrumentos que comparam o mesmo grupo no tempo, visando avaliar mudança no curso da condição estudada ou visando tomar decisões clínicas, precisam alcançar consistência interna indicada pelo alfa de Cronbach correspondente a 0,90 ou a um valor que varie, pelo menos, entre 0,50 e 0,70 quando são feitas comparações entre medidas sucessivas ou entre grupos (Ware, 1987). O alfa de Cronbach alcançado pelos itens na segunda medida de depressão do mesmo grupo de 310 idosos foi de 0,82 , sugerindo confiabilidade satisfatória.

Foi testada a consistência interna dos três fatores da CES-D. O primeiro fator ("afetos negativos") demonstrou confiabilidade intermediária $(\alpha=0,77)$ e os demais demonstraram confiabilidade baixa, sugerindo que não devem ser usados como escalas independentes, pois seus resultados ganham sentido apenas no contexto do construto depressão. Como evidência adicional de confiabilidade da CES-D, a análise da prevalência de sintomas depressivos entre as duas medidas não demonstrou diferença significativa (cerca de 34\%). Tal fato é sugestivo de que a CES-D foi capaz de reproduzir a prevalência de sintomas depressivos entre idosos residentes na comunidade.

O item 4 da CES-D foi o que demonstrou menor correlação com os demais itens. Sua retirada eleva a consistência do fator 3 ("afetos 
positivos"), tornando sua confiabilidade intermediária $(\alpha=0,61)$. O teste de Wald, realizado no contexto dos procedimentos de análise fatorial confirmatória, também indicou que a retirada do item 4 não afetaria significativamente a estrutura fatorial da escala. No estudo de Silveira e Jorge (2000) esse item também não demonstrou bom funcionamento, visto que, juntamente com o item 2 , teve as menores correlações com os demais. É provável que a tradução desse item para o português não tenha alcançado equivalência semântica ou cultural, o que teria gerado dificuldades para respondê-lo. Investigações futuras devem incluir nova redação desse item, de forma a melhorar seu funcionamento.

\section{Conclusão e considerações finais}

A versão brasileira da CES-D revelou bons índices de consistência interna e de validade de construto, assim como mostrou reprodutibilidade teste-reteste suficiente para uso como instrumento de rastreio de depressão em idosos brasileiros recrutados na comunidade. Novos estudos que comparem dados obtidos a partir dela com avaliações clínicas poderão esclarecer seu valor no diagnóstico de depressão maior nessa população. Também são necessários novos estudos com diferentes populações idosas, tais como as formadas por indivíduos residentes em asilos, hospitalizados e atendidos em ambulatórios de geriatria, para que se conheçam quais são os valores de referência mais válidos para o instrumento e possíveis variações da estrutura fatorial.

\section{Referências}

Almeida, O. P. \& Almeida, S. A. (1999). Confiabilidade da versão brasileira da escala de depressão em geriatria. Arquivos de Neuropsiquiatria, 57(2B), 421-426.

Batistoni, S. S. T., Neri, A. L. \& Cupertino, A. P. F. B. (2007). Validade da escala de depressão do Center for Epidemiological Studies (CES-D) entre idosos brasileiros. Revista Saúde Pública, 41(4), 598-605.

Beck, A. T., Ward, C. H., Mendelson, M., Mock, J. \& Erbaugh, J. (1961). An inventory for measuring depression. Archives of General Psychiatry, 4, 561-571.

Blazer, D. G. (2003). Depression in late life: Review and commentary. Journals of
Gerontology, Series A: Biological Sciences, 58(3), 249-265.

Chapleski, E., Lamphere, J., Kaczynski, R., Lichtenberg, P. \& Dwyer, J. (1997). Structure of depression measure among American Indian elders: Confirmatory factor analysis of the CES-D Scale. Research on Aging, 19(4), 462-485.

Chiriboga, D. A., Jang, Y., Banks, S. \& Kim, G. (2007). Acculturation and its effect on depressive symptom structure in a sample of Mexican American elders. Hispanic Journal of Behavioral Sciences, 29(1), 83-100.

Clark. L. A. \& Watson, D. (1991). Tripartite model of anxiety and depression: Psychometric evidence and taxonomic implications. Journal of Abnormal Psychology, 100(3), 316-336.

Dahlstro, W. M. \& Welsh, G. S. (1960). An MMPI bandbook: A guide to use in clinical practice and research. Minneapolis: University of Minnesota Press.

Davey, A., Halverson Jr. C. F., Zonderman, A. B. \& Costa Jr., P. T. (2004). Change in depressive symptoms in the Baltimore longitudinal study of aging. The Journals of Gerontology B: Psychological and Social Sciences, 59(6), P270P277.

Gatz, M., Kasl-Godley, J. E. \& Karel, M. J. (1996). Aging and mental disorders. Em J. E. Birren \& K. W. Schaie (Orgs.). Handbook of the Psychology of Aging (4 ${ }^{\mathrm{a}}$ ed.). San Diego: Academic Press.

Krause, N., Liang, J. \& Yatomi, N. (1989). Satisfaction with social support and depressive symptoms: A panel analysis. Psychology and Aging, 4(1), 88-97.

Maj, M. \& Sartorius, N. (2005). Transtornos depressivos. Porto Alegre: Artmed.

Radlof, L. S. (1977). The CES-D Scale: A selfreport depressive scale for research in the general population. Journal of Applied Psychological Measurement, 1, 385-401.

Raskin, A., Schulterbrandt, J. \& Reating, N. (1967). Factors of psychopathology in interview, ward behavior and self-report ratings of hospitalized depressives. Journal of Consultant Psychology, 31, 270.

Reifler, B. V. (1994). Depression: Diagnosis and comorbidity. Em L. S. Schneider, C. F. 
Reynolds, B. D. Lebowitz \& A. J. Friedhoff (Orgs.). Diagnosis and treatment of depression in late life. Results of the NIH consensus development conference. Washington, DC: American Psychiatric Press.

Reynolds, C. F. I. (1994). Treatment of depression in later-life. American Journal of Medicine, Suppl. 6 A, 39-46.

Silveira, D. X. \& Jorge, M. R. (2000). Escala de Rastreamento Populacional para Depressão CES-D em populações clínicas e não clínicas de adolescentes e adultos jovens. Em C. Gorestain, L. H. S. G. Andrade \& A. W. Zuarde (Orgs.). Escalas de avaliação clínica em psiquiatria e farmacologia (pp. 125-135). São Paulo: Lemos.

Ware, J. E. J. (1987). Standards for validating health measures: Definition and content.
Journal of Chronic Diseases, 40, 473-480.

Williamson, G. M. \& Schulz, R. (1992). Pain, activity restriction, and symptoms of depression among community-residing elderly adults. Journals of Gerontology, 47, 367-372.

Yesavage, J., Brink, T. L., Rose, T., Lum, O., Huang, O., Adey, V. \& Leirer, V. (1983). Development and Validation of a geriatric depression screening scale: A preliminary report. Journal of Psychiatric Research, 17, 3749.

Zich, J. M., Atkinson, C. C. \& Greenfield, T. K. (1990). Screening for depression in primary care clinics: the CES-D and the BDI. International Journal of Psychiatry Medicine, 20, 259-277.

Zung, W. W. K. (1965). A self-rating depression scale. Archives of General Psychiatry, 12, 63-79.

Recebido em julho de 2008

Reformulado em novembro de 2009 Aprovado em dezembro de 2009

Sobre as autoras:

Samila Sathler Tavares Batistoni é psicóloga, mestre em Gerontologia pela UNICAMP e doutora em Educação pela UNICAMP. Atua como professora doutora no curso de graduação em Gerontologia da EACH-USP, onde ensina e pesquisa sobre psicologia do envelhecimento, práticas psicológicas com idosos, mecanismos de autorregulação do self e saúde emocional na velhice.

Anita Liberalesso Néri é psicóloga, mestre e doutora em Psicologia pela USP, livre-docente e professora titular na Faculdade de Educação da Unicamp. Interesses em pesquisa nos campos da Psicologia do Envelhecimento e da Gerontologia incluem: bem-estar psicológico, mecanismos de autorregulação do self, atitudes em relação à velhice e paradigma lifespan.

Ana Paula Cupertino é psicóloga, mestre em Psicologia pela UNB, doutora em Desenvolvimento Humano (University of California, Davis), pós-doutoranda em Saúde Pública e Medicina Preventiva na University of Kansas Medical Center, onde ensina e pesquisa sobre socioeconomic status, life course, aging, cardiovsacular disease, overall mortality. 